\title{
SOME COMMON FIXED POINT THEOREMS IN FUZZY METRIC SPACES USING COMPATIBLE OF TYPE A-1 AND A-2
}

\author{
C. T. Aage *, J. N. Salunke \\ Department of Mathematics, North Maharashtra University, Jalgaon. \\ *Corresponding address: caage17@gmail.com \\ Received 3 August, 2009; Revised 16 February, 2010
}

\begin{abstract}
Recently M. S Khan, H. K. Pathak and R. George [9] have been introduced the concept of compatible of type A -1 and type $\mathrm{A}-2$ and obtained some common fixed point theorems in fuzzy metric spaces. In this connection we have proved some common fixed point theorems in fuzzy metric space using semicompatible mappings.
\end{abstract}

\section{INTRODUCTION}

The concept of fuzzy set was introduced by Zadeh [20]. Deng [2], Erceg [4], Kaleva [13], Kramosil and Michalek [10] built the fuzzy metric spaces in various ways. George and Veermani [5] modified the notion of fuzzy metrics spaces introduced by Kramosil and Michalek [10] in order to get a Hausdorff topology. Grebiec [6] proved the fixed point theorems for Banach contraction mappings in fuzzy metric spaces and Edelstein [3] proved it in fuzzy metric space introduced by Kramosil and Michalek [10]. In the literature the number of authors investigated the fixed point theorems for the mappings satisfying different types of contractive condition in fuzzy metric spaces.

The notion of semicompability was introduced by the Cho et al. [1] in d-topological space. They defined as a pair of self mappings $P, Q$ to be semicompatible if the conditions $(i) P x=Q x$ implies that $P Q x=Q P x(i i) P x_{n} \rightarrow x, Q x_{n} \rightarrow x$ implies that $P Q x_{n} \rightarrow Q x$ as $n \rightarrow \infty$ holds. However, in fuzzy metric spaces (ii) implies $(i)$ taking $x_{n}=y$ for all $n$ and $x=P y=Q y$. So, we can use a semicompatible pair of self-maps in fuzzy metric space by condition (ii) only. Bijendra Singh and S. Jain [18] using the semicompability proved some common fixed point theorems $[18,16,17]$ in fuzzy metric space, Menger space and D-metric space. Bijendra and S. Jain [18] discussed the relation of compatible mappings to weak compatible mappings and weak compatible mappings to semicompatible mappings in fuzzy metric space.

\section{PRELIMINARIES}

Definition 2.1 [14] A binary operation $*:[0,1] \times[0,1] \rightarrow[0,1]$ is a continuous t-norms if

* is satisfying conditions:

(i) * is an commutative and associative;

(ii) $*$ is continuous;

(iii) $a * 1=a$, for all $a \in[0,1]$; 
(iv) $a * b \leq c * d$ whenever $a \leq c, b \leq d$ and $a, b, c, d \in[0,1] \mathrm{a}, \mathrm{b}, \mathrm{c}, \mathrm{d} 2[0,1]$.

Examples of t-norms are $a * b=\min \{a, b\}$ and $a * b=a b$.

Definition 2.2 [10] The triplet $(X, M, *)$ is a fuzzy metric space if $X$ is an arbitrary set, $*$ is a continuous t- norm, $M$ is a fuzzy set in $X^{2} \times[0,1]$ satisfying the following condition $(i) M(x, y, 0)=0$.

(ii) $M(x, y, 1)=1, t>0$ if and only if $x=y$.

(iii) $M(x, y, t)=M(y, x, t)$.

(iv) $M(x, y, t) * M(y, z, s) \leq M(x, z, t+s), x, y, z \in X$ and $t, s>0$.

(v) $M(x, y, \cdot):[0, \infty) \rightarrow[0,1]$ is left continuous.

(vi) $\lim _{n \rightarrow \infty} M(x, y, t)=1$, for all $x, y \in X$.

Definition 2.3 [10] A sequence $\left\{x_{n}\right\}$ in a fuzzy metric space $M(x, y, *)$ is called Cauchy if $\lim _{n \rightarrow \infty} M\left(x_{n+p}, x_{n}, t\right)=1$ for every $t>0$ and each $p>0$.

Definition 2.4 [10] A sequence $\left\{x_{n}\right\}$ in a fuzzy metric space $M(x, y, *)$ is said to be convergent to $x \in X$ if $\lim _{n \rightarrow \infty} M\left(x_{n}, x, t\right)=1$, for each $t>0$.

Definition 2.5 [10] A fuzzy metric space $M(x, y, *)$ is said to be complete if every Cauchy sequence in $X$ converges in $X$.

Definition 2.6 [11] Two mappings $S$ and $T$ of a fuzzy metric space $M(x, y, *)$ into itself are said to be weakly commuting if $M(S T x, T S x, t) \geq M(S x, T x, t)$, for every $x \in X$.

The notion of weak commutativity is extended to R-weak commutativity by Vasuki [19] as

Definition 2.7 The mappings $S$ and $T$ of a fuzzy metric space $M(x, y, *)$ into itself are $R$-weakly commuting provided there exists some positive real number $R$ such that $M(S T x, T S x, t) \geq M\left(S x, T x, \frac{t}{R}\right)$, for all $x \in X$. Weak commutativity implies $R$-weak commutativity and the converse is true for $R=1$.

Definition 2.8 [1] Two self mappings $S$ and $T$ of a fuzzy metric space $M(x, y, *)$ are called compatible if $\lim _{n \rightarrow \infty} M\left(S T x_{n}, T S x_{n}, t\right)=1$ whenever $\left\{x_{n}\right\}$ is a sequence in $X$ such that $\lim _{n \rightarrow \infty} S x_{n}=\lim _{n \rightarrow \infty} T x_{n}=x$ for some $x \in X$. 
Definition 2.9. Let $S$ and $T$ be self maps of an FM-space $X$. The mappings $S$ and $T$ are said to be compatible of type (A) $\lim _{n \rightarrow \infty} M\left(S T x_{n}, T T x_{n}, t\right)=1$ $\lim _{n \rightarrow \infty} M\left(T S x_{n}, S S x_{n}, t\right)=1$, whenever $\left\{x_{n}\right\}$ is a sequence in $X$ such that $\lim _{n \rightarrow \infty} S x_{n}=\lim _{n \rightarrow \infty} T x_{n}=x$ for some $x \in X$.

Lemma 2.10 [15] Let $M(x, y, *)$ be a fuzzy metric space. If there exists $q \in(0,1)$ such that $M(x, y, q t) \geq M(x, y, t)$ for all $x, y \in X$ and $t>0$, then $x=y$.

Definition 2.11 [1] Let $S$ and $T$ be pair of maps of a fuzzy metric space $M(x, y, *)$. The mappings are said to be semicompatible if $\lim _{n \rightarrow \infty} M\left(S T x_{n}, T x, t\right)=1$, for all $t>0$. whenever $\left\{x_{n}\right\}$ is a sequence in $X$ such that $\lim _{n \rightarrow \infty} S x_{n}=\lim _{n \rightarrow \infty} T x_{n}=x$ for some $x \in X$.

Definition 2.12 [8] Let $S$ and $T$ be pair of maps of a fuzzy metric space $M(x, y, *)$. The mappings are said to be weak compatible if they commute at the coincidence points, that is, $S x=T x$ implies that $S T x=T S x$

Clearly if $(S, T)$ is semicompatible and $S x=T x$, then $S T x=T S x$. Therefore semicompatible implies the weak compatible but converse is not true.

Proposition 2.13 [16] Let $M(x, y, *)$ be a fuzzy metric space. Let $S, T$ be pair of compatible mappings on it, then they are weak compatible.

The converse of Proposition is not true.

Proposition 2.14 [18] Let $S$ and $T$ be self mapping on fuzzy metric space $M(x, y, *)$. If $T$ is continuous, then $(S, T)$ is semicompatible if and only if $(S, T)$ is compatible.

M. S. Khan, H. K. Pathak and R. George [9] introduced following concepts

Definition 2.15 [9] Let $S$ and $T$ be self mapping on fuzzy metric space $X$. The pair of mappings $(S, T)$ are said to be compatible of type $(A-1)$ if $\lim _{n \rightarrow \infty} M\left(S T x_{n}, T T x_{n}, t\right)=1$ whenever $\left\{x_{n}\right\}$ is a sequence in $X$ such that $\lim _{n \rightarrow \infty} S x_{n}=\lim _{n \rightarrow \infty} T x_{n}=z$ for some $z \in X$.

Definition 2.16 [9] Let $S$ and $T$ be self mapping on fuzzy metric space $X$. The pair of mappings $(S, T)$ are said to be compatible of type $(A-2)$ if 
$\lim _{n \rightarrow \infty} M\left(S T x_{n}, S S x_{n}, t\right)=1$ whenever $\quad\left\{x_{n}\right\} \quad$ is a sequence in $X$ such that $\lim _{n \rightarrow \infty} S x_{n}=\lim _{n \rightarrow \infty} T x_{n}=z$ for some $z \in X$.

Clearly, a pair $(S, T)$ of compatible type of $A$ is compatible of type $(A-1)$ and type $(A-2)$. Also a pair $(S, T)$ of compatible of type $(A-1)$ then the pair $(T, S)$ is compatible of type $(A-2)$.

Proposition 2.17 [9] Let $S$ and $T$ be self mappings of a fuzzy metric space.

(i) If $T$ is continuous then the pair of mappings $(S, T)$ is compatible of type $(A-1)$ if an only if $S$ and $T$ are compatible.

(ii) If $S$ is continuous then the pair of mappings $(S, T)$ is compatible of type $(A-2)$ if an only if $S$ and $T$ are compatible.

Proposition 2.18 Let $S$ and $T$ be self mappings of a fuzzy metric space.

(i) If $T$ is continuous then the pair of mappings $(S, T)$ is compatible of type $(A-1)$ if an only if $S$ and $T$ are semicompatible.

(ii) If $S$ and $T$ is continuous then the pair of mappings $(S, T)$ is compatible of type $(A-2)$ if an only if $S$ and $T$ are semicompatible.

Proof: (i) Let $\lim _{n \rightarrow \infty} S x_{n}=\lim _{n \rightarrow \infty} T x_{n}=z$ for some $z \in X$ and the pair is compatible of type $(A-1)$. Since $T$ is continuous we have $\lim _{n \rightarrow \infty} T T x_{n}=T z$. Therefore it follows that $\lim _{n \rightarrow \infty} M\left(S T x_{n}, T z, t\right) \geq \lim _{n \rightarrow \infty} M\left(S T x_{n}, T T x_{n}, \frac{t}{2}\right) * \lim _{n \rightarrow \infty} M\left(T T x_{n}, T z, \frac{t}{2}\right)$. That yield $\lim _{n \rightarrow \infty} M\left(S T x_{n}, T z, t\right) \geq 1 * 1=1$ and hence the pair $(S, T)$ of mappings is semicompatible Now suppose that $S$ and $T$ are semicompatible. Then $\lim _{n \rightarrow \infty} M\left(S T x_{n}, T T x_{n}, t\right) \geq \lim _{n \rightarrow \infty} M\left(S T x_{n}, T z, \frac{t}{2}\right) * \lim _{n \rightarrow \infty} M\left(T T x_{n}, T z, \frac{t}{2}\right)=1 * 1=1$,

so the pair of mappings $(S, T)$ is compatible of type $(A-1)$.

(ii) Let $\lim _{n \rightarrow \infty} S x_{n}=\lim _{n \rightarrow \infty} T x_{n}=z$ for some $z \in X$ and the pair is compatible of type $(A-2)$. Since $S$ and $T$ are continuous we have $\lim _{n \rightarrow \infty} S T x_{n}=S z, \lim _{n \rightarrow \infty} S S x_{n}=S z, \lim _{n \rightarrow \infty} T S x_{n}=T z$. Therefore it follows that

$$
\begin{aligned}
M\left(S T x_{n}, T z, t\right) & \geq M\left(S T x_{n}, T S x_{n}, \frac{t}{2}\right) * M\left(T S x_{n}, T z, \frac{t}{2}\right) \\
& \geq M\left(S T x_{n}, S S x_{n}, \frac{t}{4}\right) * M\left(S S x_{n}, T z, \frac{t}{4}\right) * M\left(T S x_{n}, T z, \frac{t}{2}\right) \\
& =1 * 1 * 1=1 .
\end{aligned}
$$


That yield $M\left(S T x_{n}, T z, t\right) \geq 1 * 1 * 1=1$ and hence the pair $(S, T)$ of mappings is semicompatible.

Now suppose that $S$ and $T$ are semicompatible. Then we have

$$
\begin{aligned}
M\left(S T x_{n}, S S x_{n}, t\right) & \geq M\left(S T x_{n}, T z, \frac{t}{2}\right) * M\left(T z, S S x_{n}, \frac{t}{2}\right) \\
& \geq M\left(S T x_{n}, T z, \frac{t}{2}\right) * M\left(T z, S T x_{n}, \frac{t}{4}\right) * M\left(S T x_{n}, S S x_{n}, \frac{t}{4}\right) \\
& =1 * 1 * 1=1 .
\end{aligned}
$$

So the pair $(S, T)$ is compatible of type $(A-2)$.

Example 2.19 Let $X=[0,1]$ and let $(X, M, *)$ be a fuzzy metric space with $M(x, y, t)=\left[\frac{\exp |x-y|}{t}\right]^{-1}$, for all $x, y \in X, t>0$. Define mapping as follows

$$
S x=\left\{\begin{array}{l}
x \text { if } 0 \leq x \leq \frac{1}{2} \\
1 \text { if } \quad x \geq \frac{1}{2}
\end{array}\right.
$$

Let $T$ be an identity mapping on $X$ and $x_{n}=\frac{1}{2}-\frac{1}{n}$. Then $\left\{S x_{n}\right\}=\left\{\frac{1}{2}-\frac{1}{n}\right\} \rightarrow \frac{1}{2}$, $\left\{\operatorname{Tx}_{n}\right\}=\left\{\frac{1}{2}-\frac{1}{n}\right\} \rightarrow \frac{1}{2},\left\{S T x_{n}\right\}=\left\{T x_{n}\right\}=\left\{\frac{1}{2}-\frac{1}{n}\right\} \rightarrow \frac{1}{2},\left\{T S x_{n}\right\}=\left\{S x_{n}\right\}=\left\{\frac{1}{2}-\frac{1}{n}\right\} \rightarrow \frac{1}{2}$, $\left\{S S x_{n}\right\}=\left\{S x_{n}\right\}=\left\{\frac{1}{2}-\frac{1}{n}\right\} \rightarrow \frac{1}{2}, T z=\frac{1}{2}$, so clearly the both pairs (S, T) and (T, S) are compatible of type $(\mathrm{A}-1)$ and compatible of type $(\mathrm{A}-2)$. $\left\{S T x_{n}\right\}=\left\{S x_{n}\right\}=\left\{\frac{1}{2}-\frac{1}{n}\right\} \rightarrow \frac{1}{2}=T\left(\frac{1}{2}\right) . \quad$ So $\quad(S, T) \quad$ is $\quad$ semicompatible but $\left\{T S x_{n}\right\}=\left\{S x_{n}\right\} \rightarrow \frac{1}{2} \neq S\left(\frac{1}{2}\right)$. So $(T, S)$ is not semicompatible.

Proposition 2.20 Let $S$ and $T$ be self maps of a fuzzy metric space $X$. If the pair $(S, T)$ is semicompatible and $S z=T z$ for some $z$ in $X$ then $z$ is common fixed point of $S$ and $T$.

Proof: Let $\left\{x_{n}\right\}$ be a sequence in $X$ defined by $x_{n}=z$ for $n=1,2, \cdots$ and let $S z=T z$. Then we have $\lim _{n \rightarrow \infty} S x_{n}=S z$ and $\lim _{n \rightarrow \infty} T x_{n}=T z$. Since the pair (S, T) is semicompatible, we have $1=\lim _{n \rightarrow \infty} M\left(S T x_{n}, T z, t\right)=M\left(S T x_{n}, S z, t\right)$ implies that $S T z=S z$ i.e. $T z=z=S z$, hence $z$ is common fixed point of $S$ and $T$.

\section{RESULTS}


Lemma 3.1 Let $(X, M, *)$ be a fuzzy metric space with $t * t \geq t$ for all $t \subset[0,1]$ and $P, Q, S, T$ be self maps of $X$ such that

(i) $P(X) \subset T(X)$ and $Q(X) \subset S(X)$

$$
\begin{aligned}
{[1+p M(S x, T y, k t)] * M(P x, Q y, k t) \geq p } & {\left[\begin{array}{c}
M(P x, S x, k t) * M(Q y, T y, k t) \\
+M(P x, T y, k t) * M(Q y, S x, k t)
\end{array}\right] } \\
& +M(S x, T y, t) * M(P x, S x, t) * M(Q y, T y, t) \\
& * M(P x, T y, t) * M(Q y, S x,(2-\alpha) t)
\end{aligned}
$$

for all $x, y \in X, p \geq 0$ and $\alpha \in(0,2)$ and $k \in(0,1)$. Then $X$ contains a Cauchy sequence.

Proof: The proof is given in [9].

Theorem 3.2 Let $(X, M, *)$ be a complete fuzzy metric space with $t * t \geq t$ for all $t \subset[0,1]$

and let $P, Q, S$ and $T$ be self mappings of $X$ satisfying $(i)$ and $(i i)$ of Lemma 3.1 and the following:

(iii) the pairs $(P, S)$ and $(Q, T)$ are semicompatible,

(iv) one of $S$ or $T$ is continuous.

Then $P, Q, S$ and $T$ have a unique common fixed point in $X$.

Proof: Let $x_{0}$ be a arbitrary point in $X$. Choose $x_{1} \in X$ such that $P x_{0}=T x_{1}$ and for $x_{1}$ we choose $x_{2} \in X$ such that $S x_{2}=Q x_{1}$. Continuing in this way we get a sequence $y_{n}$ as follow

$$
y_{2 n}=P x_{2 n}=T x_{2 n+1} \text { and } y_{2 n+1}=Q x_{2 n+1}=S x_{2 n+2}
$$

By Lemma 3.1, $y_{n}$ is a Cauchy sequence in $X$. Since $X$ is complete, so it should converges to some point $z$ in $X$. Therefore the subsequences $P x_{2 n}, T x_{2 n+1}, Q x_{2 n+1}$ and $S x_{2 n+2}$ also converges to $z$. Suppose $T$ is continuous, then $T T x_{2 n+1} \rightarrow T z$, as $n \rightarrow \infty$. Since the pair $(Q, T)$ is semicompatible, then $\lim _{n \rightarrow \infty} Q T y_{n}=T z$. For $\alpha=1$, in $(i i)$, we put $x=x_{2 n}, y=T x_{2 n+1}$, then 


$$
\begin{aligned}
& {\left[1+p M\left(S x_{2 n}, T T x_{2 n+1}, k t\right)\right] * M\left(P x_{2 n}, Q T x_{2 n+1}, k t\right) \geq} \\
& p\left[\begin{array}{l}
M\left(P x_{2 n}, S x_{2 n}, k t\right) * M\left(Q T x_{2 n+1}, T T x_{2 n+1}, k t\right) \\
+M\left(P x_{2 n}, T T x_{2 n+1}, k t\right) * M\left(Q T x_{2 n+1}, S x_{2 n}, k t\right)
\end{array}\right] \\
& +M\left(S x_{2 n}, T T x_{2 n}, t\right) * M\left(P x_{2 n}, S x_{2 n}, t\right) * M\left(Q T x_{2 n+1}, T T x_{2 n+1}, t\right) \\
& \text { *M(Px} \left.x_{2 n}, T T x_{2 n+1}, t\right) * M\left(Q T x_{2 n+1}, S x_{2 n}, t\right)
\end{aligned}
$$

implies $\quad M(z, T z, k t) \geq M(z, T z, t) * 1 * 1 * M(z, T z, t) * M(T z, z, t)$. This shows that $T z=z$. Aging from (ii) for $\alpha=1$, we have

$$
\begin{gathered}
{\left[1+p M\left(S x_{2 n}, T z, k t\right)\right] * M\left(P x_{2 n}, Q z, k t\right) \geq p\left[\begin{array}{l}
M\left(P x_{2 n}, S x_{2 n}, k t\right) * M(Q z, T z, k t) \\
+M\left(P x_{2 n}, T z, k t\right) * M\left(Q z, S x_{2 n}, k t\right)
\end{array}\right]} \\
+M\left(S x_{2 n}, T z, t\right) * M\left(P x_{2 n}, S x_{2 n}, t\right) * M(Q z, T z, t) \\
* M\left(P x_{2 n}, T z, t\right) * M\left(Q z, S x_{2 n}, t\right)
\end{gathered}
$$

which gives $M(z, Q z, k t) \geq 1 * 1 * M(Q z, z, t) * 1 * 1 * M(Q z, z, t)$ and hence $Q z=z$, therefore by (i) there exist $u \in X$ such that $Q z=S u$ and by (ii) for $\alpha=1$, we have

$$
\begin{aligned}
{[1+p M(S u, T z, k t)] * M(P u, Q z, k t) \geq } & p\left[\begin{array}{l}
M(P u, S u, k t) * M(Q z, T z, k t) \\
+M(P u, T z, k t) * M(Q z, S u, k t)
\end{array}\right] \\
& +M(S u, T z, t) * M(P u, S u, t) * M(Q z, T z, t) \\
& * M(P u, T z, t) * M(Q z, S u, t)
\end{aligned}
$$

which gives $M(P u, z, k t) \geq 1 * M(P u, z, k t) * 1 * M(P u, z, t) * 1$ and hence $P u=z$. Therefore $P u=S u=z$ and since the pair $(P, S)$ is semicompatible then we have

$P S u=S u$ and so $P z=z$. Now we claim that $S z=z$. From (ii) we have

$$
\begin{aligned}
{[1+p M(S z, T z, k t)] * M(P z, Q z, k t) \geq p } & {\left[\begin{array}{r}
M(P z, S z, k t) * M(Q z, T z, k t) \\
+M(P z, T z, k t) * M(Q z, S z, k t)
\end{array}\right] } \\
& +M(S z, T z, t) * M(P z, S z, t) * M(Q z, T z, t) \\
& * M(P z, T z, t) * M(Q z, S z, t)
\end{aligned}
$$

which gives $M(S z, z, k t) \geq M(S z, z, t) * M(z, S z, t) * 1 * 1 * M(z, S z, t)$. It gives $S z=z$. Hence $z$ is common fixed point of $P, Q, S$ and $T$. If $S$ is continuous then we get similar result as above. Uniqueness of the fixed point follows form (ii). 
Theorem 3.3 Let $(X, M, *)$ be a complete fuzzy metric space with $t * t \geq t, t \in[0,1]$ and let $P, Q, S$ and $T$ be self mappings of $X$ satisfying

(i) $P(X) \subset T(X)$ and $Q(X) \subset S(X)$

(ii)

$[1+p M(S x, T y, k t)] * M(P x, Q y, k t) \geq\left[\begin{array}{l}M(S x, T y, t) * M(P x, S x, t) * M(Q y, T y, t) \\ M(P x, T y, t) * M(Q y, S x,(2-\alpha) t)\end{array}\right]$

for all $x, y \in X, p \geq 0$ and $\alpha \in(0,2)$ and $k \in(0,1)$,

(iii) the pairs $(P, S)$ and $(Q, T)$ are semicompatible,

(iv) one of $S$ or $T$ is continuous.

Then $P, Q, S$ and $T$ have a unique common fixed point in $X$.

Proof: Put $p=0$ in Theorem 3.1, we get required result.

Theorem 3.4 Let $(X, M, *)$ be a complete fuzzy metric space with $t * t \geq t, t \in[0,1]$ and let the pair $(P, S)$ of self semicompatible mappings on $X$ satisfying

(i) $P(X) \subset S(X)$

(ii)

$$
\begin{aligned}
{[1+p M(S x, S y, k t)] * M(P x, P y, k t) \geq p } & {\left[\begin{array}{l}
M(P x, S x, k t) * M(P y, S y, k t) \\
+M(P x, S y, k t) * M(P y, S x, k t)
\end{array}\right] } \\
& +M(S x, S y, t) * M(P x, S x, t) * M(P y, S y, t) \\
& * M(P x, S y, t) * M(P y, S x,(2-\alpha) t)
\end{aligned}
$$

for all $x, y \in X, p \geq 0$ and $\alpha \in(0,2)$ and $k \in(0,1)$,

(iii) $P$ is continuous

Then $P$ and $\mathrm{S}$ have a unique common fixed point in $X$.

Proof: Put $S=T$ and $P=Q$ in Theorem 3.1, we get above result.

\section{REFERENCES}

[1] Cho Y J, Pathak H K, Kang S M \& Jung J S, Common fixed points of compatible maps of type $\beta$ on fuzzy metric spaces, Fuzzy Sets and Systems, 93 (1998),99.

[2] Deng Z K, Fuzzy pseudo-metric spaces, J. Math. Anal. Appl. 86 (1982), 7495.

[3] Edelstein M, On fixed and periodic points under contraction mappings, J. London Math. Soc. 37 (1962) 7479. 
[4] Erceg M A, Metric spaces in fuzzy set theory, J. Math. Anal. Appl. 69 (1979), 205230.

[5] George A \& Veeramani P, On some results in fuzzy metric spaces, Fuzzy Sets and Systems, 64 (1994), 395.

[6] Grabiec M, Fixed points in fuzzy metric spaces, Fuzzy Sets and Systems 27 (1988), 385.

[7] Hadzic O, Common fixed point theorems for families of mapping in complete metric space, Math. Japon. 29 (1984), 127.

[8] Jungck G \& Rhoades B E, Fixed Point for Set Valued functions without Continuity, Indian J. Pure Appl. Math., 29(3), (1998), pp.771.

[9] Khan M S, Pathak H K \& George R, Compatible mappings of type A - 1 and type A -2 and common fixed points in fizzy metric spaces, Int. Math. Forum, 2, 2007, no. 11, 515.

[10] Kramosil O \& Michalek J, Fuzzy metric and statistical metric spaces, Kybernetica, 11 (1975), 326.

[11] Pant R P \& Jha K, A remark on common fixed points of four mappings in a fuzzy metric space, J. Fuzzy Math. 12(2) (2004), 433.

[12] Pathak H K \& Singh Prachi, Common Fixed Point Theorem for Weakly Compatible Mapping, International Mathematical Forum, 2, 2007, no. 57, 2831.

[13] Kaleva O \& Seikkala S, On fuzzy metric spaces, Fuzzy Sets and Systems, 12 (1984), 215.

[14] Schweizer B \& Sklar A, Statistical metric spaces, Pacific J. Math. 10(1960), 313.

[15] Sharma S, Common fixed point theorems in fuzzy metric spaces, Fuzzy Sets and Systems 127 (2002), 345.

[16] Singh B \& Jain S, A fixed point theorem in Menger space through weak compatibility, J. Math. Anal. Appl. 301 (2005), no. 2, 439448.

[17] Singh B \& Jain S, Weak compatibility and fixed point theorems for four selfmaps in d-metric spaces, International Journal of Mathematics and Mathematical Sciences, 15 (2005) 2473.

[18] Singh B \& Jain S, Semicompatibility and fixed point theorems in fuzzy metric space using implicit relation, International Journal of Mathematics and Mathematical Sciences, 16 (2005) 26172629. 
KATHMANDU UNIVERSITY JOURNAL OF SCIENCE, ENGINEERING AND TECHNOLOGY

VOL. 7, No. I, SEPTEMBER, 2011, pp 18-27

[19] Vasuki R, Common fixed points for R-weakly commuting maps in fuzzy metric spaces, Indian J. Pure Appl. Math. 30 (1999), 419.

[20] Zadeh L A, Fuzzy sets, Inform and Control 8 (1965), 338. 